\title{
Corrigendum: Interferon regulatory factor 9 is critical for neointima formation following vascular injury
}

Shu-Min Zhang, Li-Hua Zhu, Hou-Zao Chen, Ran Zhang, Peng Zhang, Ding-Sheng Jiang, Lu Gao, Song Tian, Lang Wang, Yan Zhang, Pi-Xiao Wang, Xiao-Fei Zhang, Xiao-Dong Zhang, De-Pei Liu \& Hongliang Li

Nature Communications 5:5160 doi: 10.1038/ncomms6160 (2014); Published 16 Oct 2014; Updated 31 Mar 2015

The value of the relative luciferase activity of RASMCs infected with AdshIRF9 and AdshSIRT1 in Supplementary Fig. 9a in this Article is incorrect; it was inadvertently duplicated from the data point representing the relative luciferase activity of RASMCs infected with AdshIRF9 alone. The correct version of Supplementary Fig. 9 appears below.

a

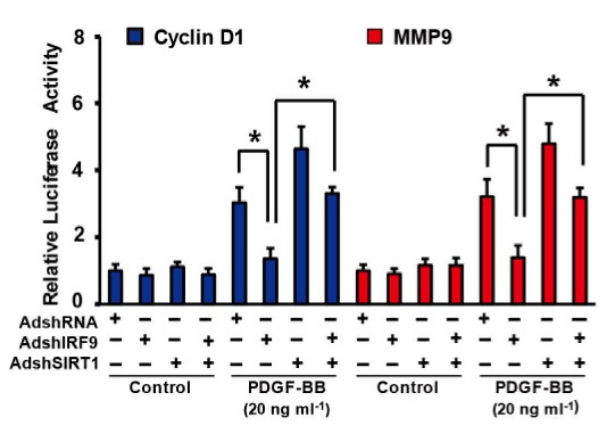

C
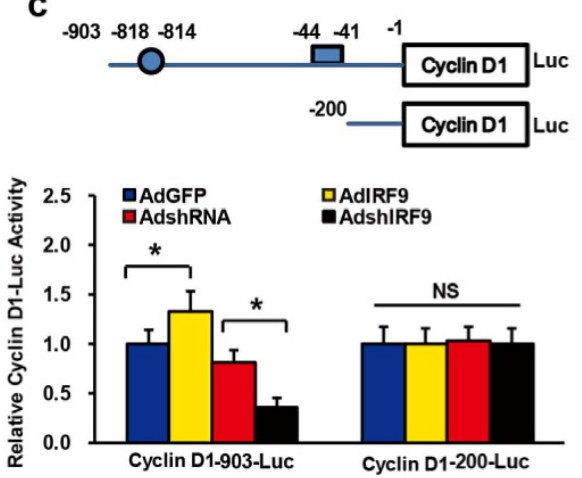

b

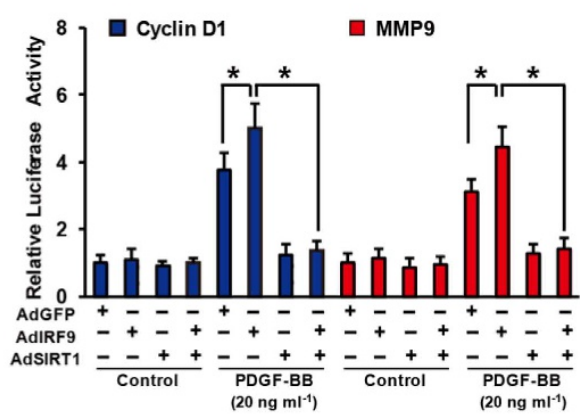

d

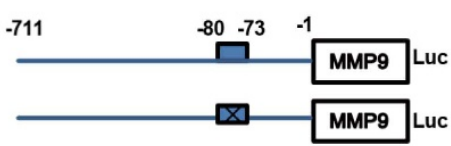

AP-1 binding site

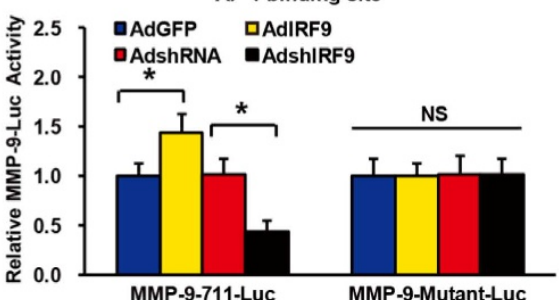

Supplementary Figure 9 